\title{
An Explanation of Subsurface Optical Pathways through Food Myosystems and their Effect on Colorimetry
}

\author{
H. J. Swatland \\ University of Guelph, Guelph \\ Ontario, Canada \\ Email: Swatland [AT] uoguelph.ca
}

\begin{abstract}
Light may pass along and across the long axes of muscle fibers in any food myosystem. Thus, incident light may be scattered in several ways before some of it reappears at the surface as diffuse reflectance. Pathways may be short if scattering is strong, or long if scattering is weak. Short pathways minimize selective absorbance by chromophores such as myoglobin, while long pathways maximize selective absorbance. Many food myosystems exhibit a post-mortem decrease in pH caused by anaerobic glycolysis with a series of microstructural changes - glycogen granules between myofibrils are lost, myofibrils shrink laterally as myofilaments move closer together, water moves from within myofibrils to the space between them, muscle fiber membranes leak and lose their electrical capacitance, and myoglobin is flushed into the fluid filled spaces between muscle fibers. These changes increase scattering of light passing across the long axes of muscle fibers. Scattering of light along muscle fibers is caused by sarcomere discs (Abands). Interference from one or a small number of sarcomere discs may cause iridescence, but in most cases interference from numerous discs causes achromatic diffuse reflectance. Commission International de l'Éclairage (CIE) chromaticity coordinates were calculated for various subsurface optical pathways. Pathways across versus along muscle fibers had a strong effect on CIE $y(r=0.84, P<0.01)$ and an even stronger effect on CIE Y\% $(r=0.95, P<$ 0.005).
\end{abstract}

Keywords - Food myosystems, Colorimetry, Optical pathways

\section{INTRODUCTION}

The color of food myosystems such as meat, poultry and fish has an obvious importance to those who purchase these foods visually but, for many years, researchers were preoccupied by the spectrophotometry of soluble pigments - purple myoglobin, bright red oxymyoglobin, and brown metmyoglobin, and only recently has light scattering been acknowledged in meat colorimetry. The early research on meat colorimetry was driven by a concern for supermarket consumers who wanted bright red oxymyoglobin beef, supported by the packaging film industry trying to limit the formation of brown metmyoglobin [1]. Paradoxically, there are now many profitable markets that espouse well aged meat - metmyoglobin brown, but superior in taste and tenderness. Almost totally ignored in early research was the fact that bright red meat turns brown when cooked - few if any new pigments are formed deep in the meat, but there are major changes in light scattering with cooking [2]. Commercially important problems such as pale, soft, exudative (PSE) pork and poultry meat and DFD (dark, firm, dry) beef had little explanation if meat color was simply caused by soluble chromophores in sarcoplasm. Paleness from high light scattering is associated with wetness (fluid loss from muscle fibers), while darkness is associated with dryness (fluid retained within muscle fibers). Hence, light scattering and fluid movement are interdependent [3].

It has been known for many years that a colorimeter placed on a cut surface of a myosystem responds to subsurface optical pathways - measurements across the grain of skeletal muscle differ from those along the grain [4]. The explanation given here is an update of an invited lecture presented in Brazil in 2011, where the primary goal was to explain things rather than to reference hundreds of research papers [5]. Many of the new ideas proposed in 2011 were contentious, but have now been adopted by other researchers $[6,7]$. If there is now a consensus that subsurface optical pathways can affect the colorimetry of food myosystems, the next step is to quantify the relationships. Original data from optical experiments were re-analyzed to calculate CIE chromaticity coordinates [8].

\section{OPTICAL PROPERTIES OF BULK MYOSYSTEMS}

Any analytical optical system such as a colorimeter, a fiber-optic probe or video image analysis (VIA) involves three major pathways. The incident light (Fig. 1, A) may be directly reflected from the myosystem surface (Fig. 1, B). This Fresnel or specular (mirror-like) reflectance is partly polarized and has virtually no selective absorbance by myoglobin. If the incident illumination is white light, then the specular reflectance is white light, just as artists give the impression of a metallic or wet surface by painting white highlights. When VIA is used to measure intramuscular adipose tissue (small 
deposits of fat in a myosystem), it is important to minimize specular reflectance using a polarizer otherwise bright spots of reflected light are difficult to distinguish from small deposits of adipose tissue. Specular reflectance follows Fresnel equations and has a strong angular dependency, but raw myosystem surfaces are seldom smooth. Some of the light that is not reflected enters below the myosystem surface, while the remainder may be scattered back to escape as diffuse Lambertian reflectance from the myosystem surface (Fig. 1, C and D). Diffuse scattered light from myosystem appears almost the same at all viewing and illumination angles, mostly following Lambert's law,

$$
\mathrm{I}=\mathrm{I}_{0} \cos \Theta
$$

where $\mathrm{I}$ is the luminous intensity, $\mathrm{I}_{0}$ is the luminous intensity in the normal direction, and $\Theta$ is the angle from the normal. But all diffuse light scattered from a myosystem is not truly Lambertian. This does not affect the performance of a typical colorimeter, but is likely to affect the results from systems using coherent light from a laser. The interpretation of laser speckle patterns is somewhat speculative compared to the classical optical pathways considered in this report.

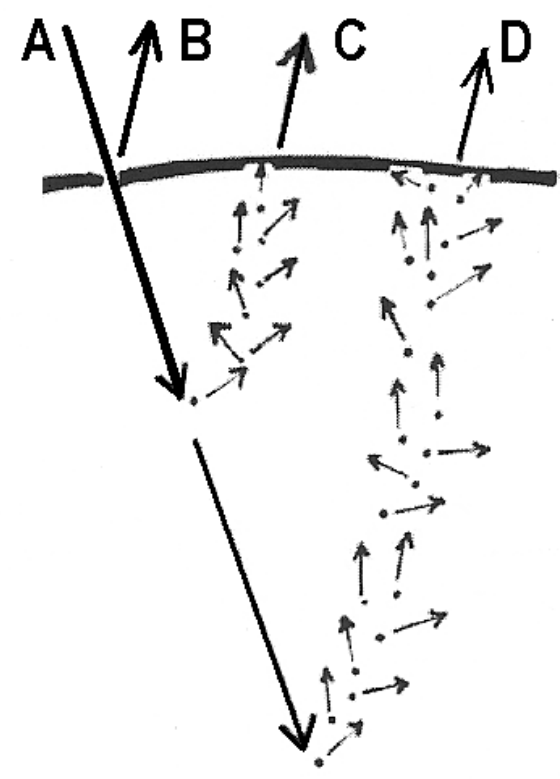

Fig.1. Light incident on a bulk myosystem (A) may be reflected at the surface (B) or scattered through short (C) or long (D) pathways.

Pathway $\mathrm{C}$ in Fig. 1 has a high degree of microstructural scattering that minimizes selective absorbance by soluble chromophores and the myosystem appears pale. But pathway D in Fig. 1 is longer, because of a low degree of microstructural scattering, thus maximizing selective absorbance by chromophores and the myosystem appears dark. Pathway C in Fig. 1 shows what happens with PSE myosystems. Pathway D in Fig. 1 shows what happens with DFD pork, beef and poultry myosystems. But changes between pathways may occur during post-mortem metabolism. Immediately after stunning and exsanguination, pathway D dominates the appearance of the myosystem - it is dark. If normal postmortem glycolysis occurs and the $p \mathrm{H}$ of the myosystem declines, then pathways change in the direction from $\mathrm{D}$ to $\mathrm{C}$, thus a system such as beef will develop a normal bright red appearance. But if glycolysis is unusually rapid (fast $p \mathrm{H}$ decline) or extensive (low ultimate $p \mathrm{H}$ ), then pathway $\mathrm{C}$ becomes dominant and the myosystem appears PSE.

Many researchers who measure the color of myosystems are oblivious of the way that the apparatus itself interacts with the sample. For example, a fiber-optic probe may use either a short or a long pathway through the myosystem, depending on the separation of its illumination and receiving apertures. And the apertures themselves may change depending on the refractive index of myosystem fluids post-mortem - their refractive index first increases (osmotic loss of interstitial fluid) and then decreases (release of water from myofibrils). Different methods give different results. If an optical system is designed to measure light scattering, then it is best to have illumination and photometry close together and at $90^{\circ}$ to the sample surface to follow pathway $\mathrm{C}$ in Fig. 1. This minimizes selective absorbance by soluble chromophores. For example, for premium pale veal where the objective is to assess low myoglobin, this pathway will detect low $\mathrm{pH}$ not myoglobin. And to detect myoglobin levels to identify calves that have obtained dietary iron, pathway D in Fig. 1 produces better results. Another example is fish such as farmed Arctic Char, where dietary astaxanthin supplements are required to produce a yellow coloration of the myosystem: pathway D in Fig. 1 produces the best result. Without any astaxanthin in the diet, the pale myosystem is 
almost moribund, which can be detected by the loss of membrane capacitance - another example of optical and electrical properties being interrelated.

In Fig. 1, the microstructural sources of scattering along pathways $\mathrm{C}$ and $\mathrm{D}$ have been simplified to random dots but, scattering really originates from the surfaces of muscle fibers and, myofibrils, from refraction of light passing through birefringent myofibrils within muscle fibers, and sometimes with an extra contribution to scattering from any proteins that have escaped from muscle fibers and been precipitated by a low $\mathrm{pH}$. Soluble sarcoplasmic proteins escape when the muscle fiber membrane loses ATP (adenosine triphosphate), which can be detected biochemically and by the loss of electrical capacitance. Without ATP, membranes stop pumping sodium ions and electrolytes inside and outside membranes become continuous so an electrical charge across membranes is eliminated and electrical capacitance is lost. Thus, optical pathways are often correlated with electrical pathways.

There are many possibilities for light passing through muscle fibers: the main features are shown in Fig. 2. At point A in Fig. 2 the incident light may be scattered in a truly random manner if sarcoplasmic proteins have precipitated on the myofibril surface. At point B in Fig. 2, Fresnel reflectance may occur on the surface of the myofibril where there is an optical boundary between the myofilament lattice (high refractive index) and the sarcoplasm (low refractive index). Myofibrils are very small and very numerous, so the reflected light is randomized in a bulk myosystem. But, on individual myofibrils seen in a polarizing microscope, the mirror-like nature of specular Fresnel reflectance is detectable. At point $\mathrm{C}$ in Fig. 2, incident light is refracted to pass through the myofibril. The angle of refraction depends on changes on refractive index caused by post-mortem glycolysis. As $p \mathrm{H}$ decreases, filaments move close together laterally and refractive index increases. Thus, at a low ultimate $p \mathrm{H}$, scattering is high and the myosystem appears pale, as in pathway $\mathrm{C}$ of Fig. 1.

Another source of scattering is by reflectance from sarcomere disks, as at point D in Fig. 2. This idea took some time to gain acceptance because the basic concept was buried in the oldest literature of muscle histology [9]. The sliding filament theory of muscle contraction is undoubtedly correct, but it caused everyone to take a lateral view of muscle fibers and myofibrils to understand the events of contraction. But many years before this, Bowman had discovered that muscle fibers sometimes fragment into discs, most likely high-refractive A bands and low refractive I bands. Thus, the surfaces of these discs are reflective and, being stacked in an orderly manner, are capable of destructive and constructive interference giving rise to metallic interference colors. Other researchers have recently adopted this hypothesis [10].

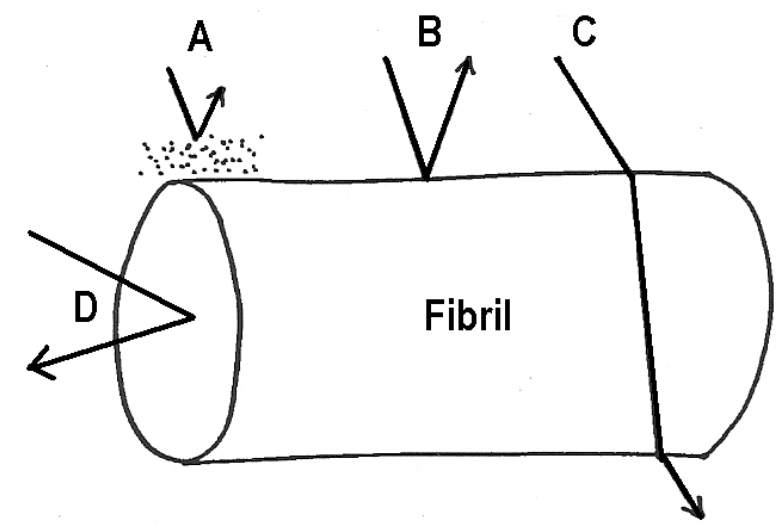

Fig.2. Myofibrillar scattering: from precipitated sarcoplasmic proteins (A), surface reflectance (B), refraction through the myofibril (C), and reflectance from sarcomere disks (D). 

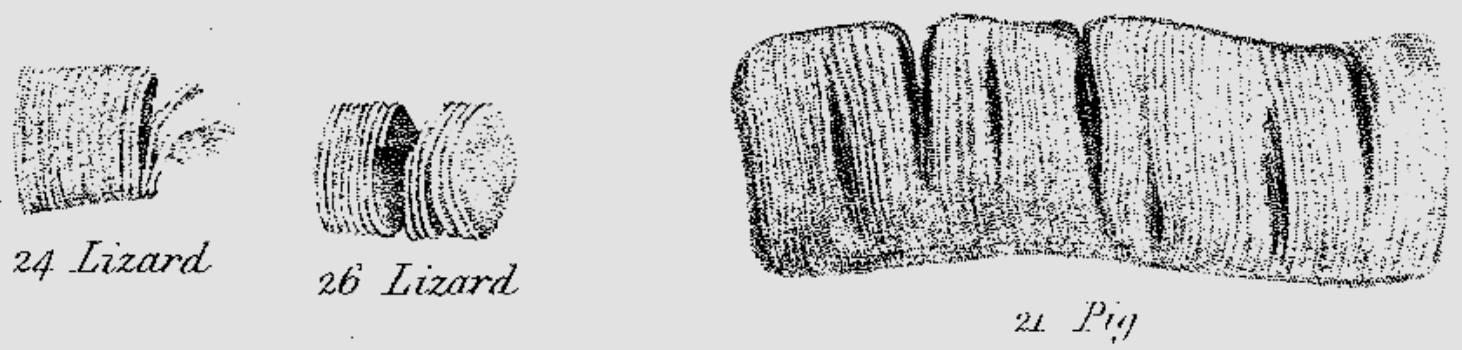

Fig. 3. Bowman's discovery in 1840 - striated skeletal muscle fibers separating into discs [9].

Refractive scattering needs explaining. In Fig. 2 it is obvious how light at points A, B and D can be scattered back to the myosystem surface, but what about the refracted light at point $\mathrm{C}$ which still looks as if it is passing deeper into the myosystem? To answer this question take into account that refracted light passes through large numbers of myofibrils. As seen in Fig. 4, an increase in the angle of refraction may send incident light back to the myosystem surface.

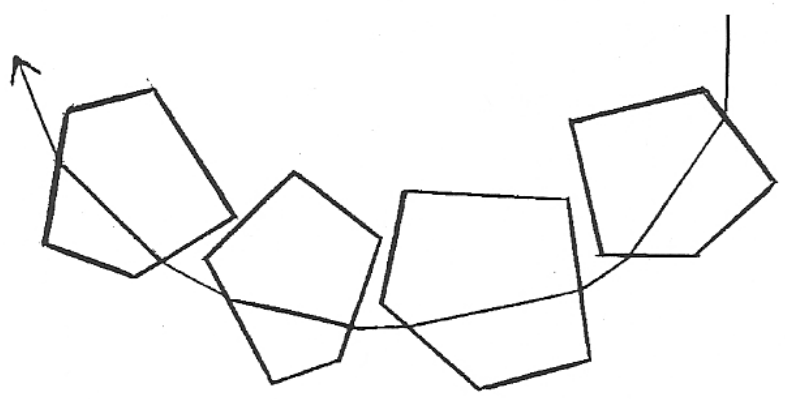

Fig. 4. Myofibrils seen in cross section, showing how refracted light may change direction to cause incident light to be sent back to the myosystem surface as Lambertian scattering.

Fig. 4 is highly diagrammatic, but Fig. 5 shows the full complexity - a transverse section of beef before post-mortem glycolysis. The glycogen in the sarcoplasm between myofibrils has been stained by the periodic-acid Schiff reaction so that myofibrils appear as clear spaces. As post-mortem glycolysis proceeds, glycogen disappears and the myofibrils decrease their diameter. Exactly the same pattern may be observed by precipitating myoglobin with sulfosalicylic acid it starts in solution between myofibrils then leaks in to the spaces between muscle fibers once the fiber membrane runs out of ATP.

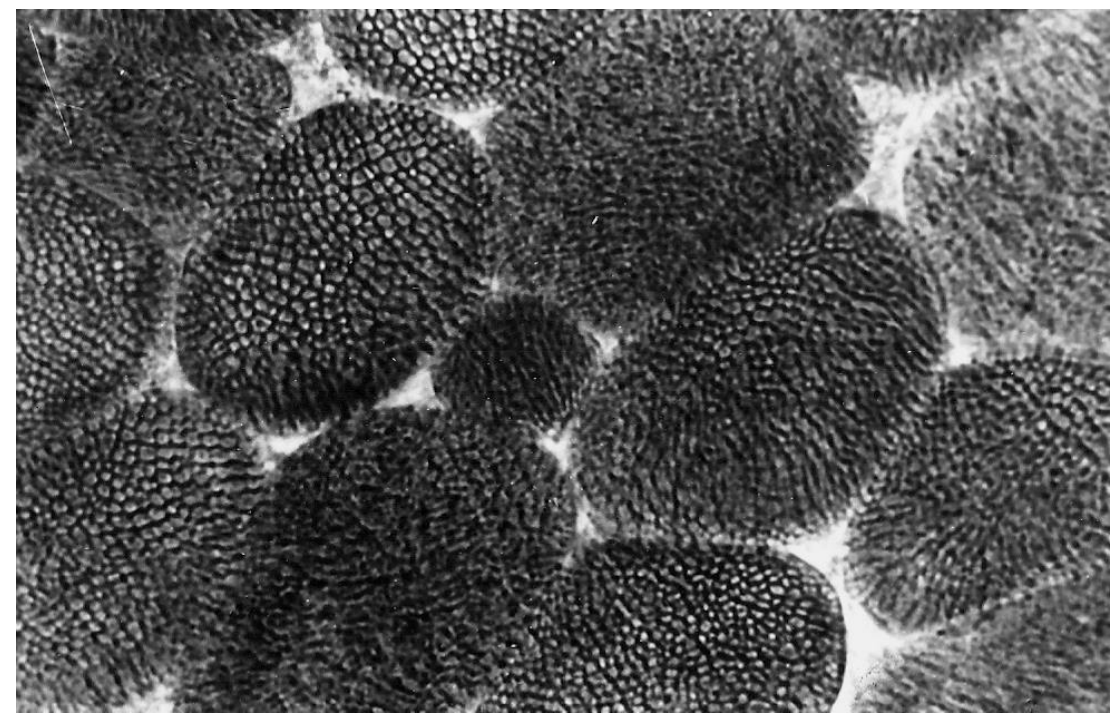


Fig. 5. Transverse section of beef muscle fibers immediately after stunning and exsanguination stained for glycogen by the periodic-acid Schiff reaction and seen by light microscopy.

As living muscle is converted to a food myosystem, a major physical change is the rigor bonding of actomyosin locking thick and thin filaments together. Filament sliding is prevented and sarcomere length from $\mathrm{Z}$ line to $\mathrm{Z}$ line becomes fixed, although it may appear to increase if tensile forces disrupt $Z$ lines as a sample is removed for examination. Long sarcomeres give a tender myosystem with high water-holding capacity (WHC), while short sarcomeres give a tough myosystem with low WHC.

WHC determines how much fluid will be released from a myosystem, often at great economic cost during refrigeration and myosystem processing. Fig. 6 shows a transverse section of pork after the completion of post-mortem glycolysis. Hence, glycogen granules have been lost, myofibrils have decreased in diameter, and much of the water from between filaments has been released. Restraints such as desmin are visible, giving some indication of the complexity of WHC. Not only is the system affected by $p \mathrm{H}$ acting directly on the lateral negative electrostatic repulsion between filaments, but also by post-mortem proteolysis of proteins linking myofibrils and, thus, affecting the separation of myofibrils as they shrink.

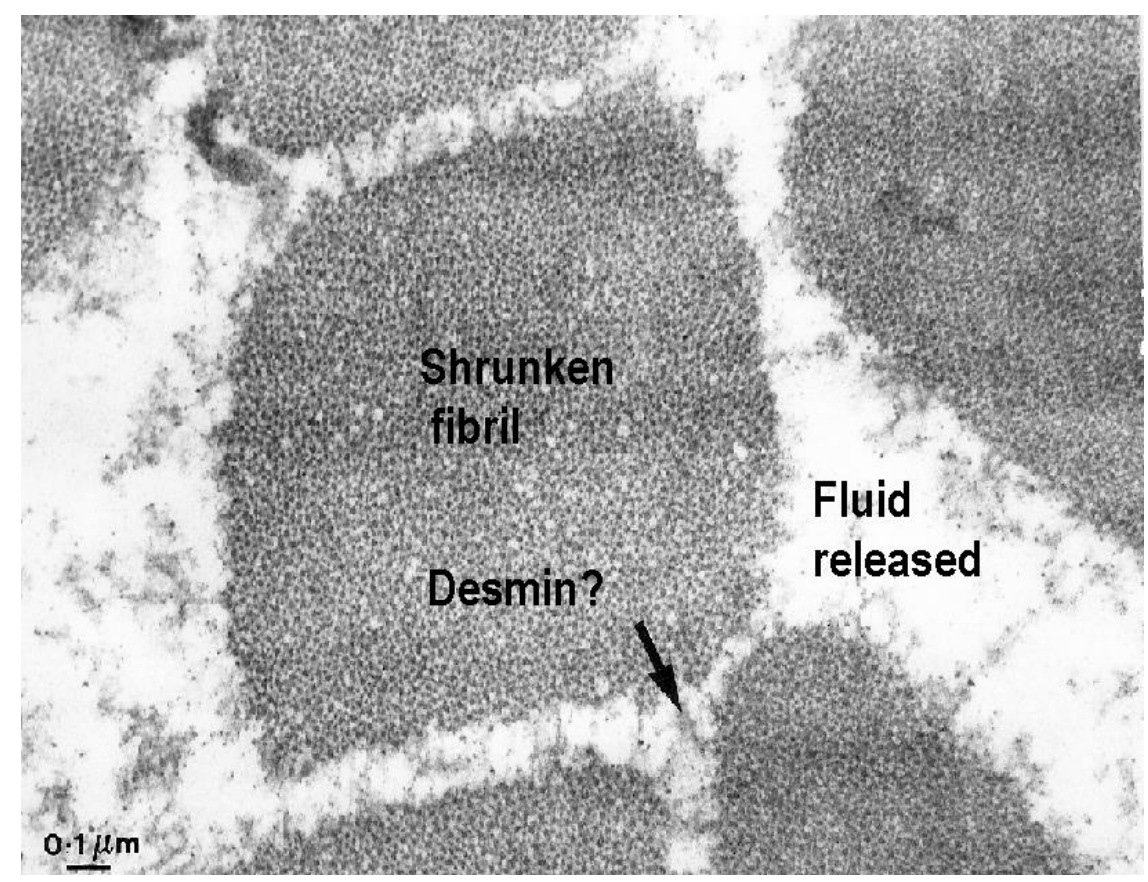

Fig. 6. Electron micrograph of pork in transverse section.

To follow the release of water from myofibrils, we may use X-ray diffraction (Fig.7). This shows that, as the $p \mathrm{H}$ approaches the isoelectric point of muscle proteins, a decrease in negative electrostatic repulsion reduces the lateral separation of filaments. This allows water to move from the filament lattice, into the sarcoplasm, through the muscle fiber membrane, into the extracellular space, and then to be lost from the myosystem as drip or evaporation - often at great commercial loss, especially in pork. Fluid movements as living muscle is converted to food myosystem can be detected electrically. On the surface of a muscle fiber there is a plasma membrane through which fluid released from the fiber will move into the interstitial (intercellular) space. Membranes in living muscle have a high dielectric constant measurable as capacitance using an alternating current. As post-mortem metabolism proceeds, and membranes leak fluid into the interstitial space, the capacitance is lost. Electrical resistance also decreases at this time. 


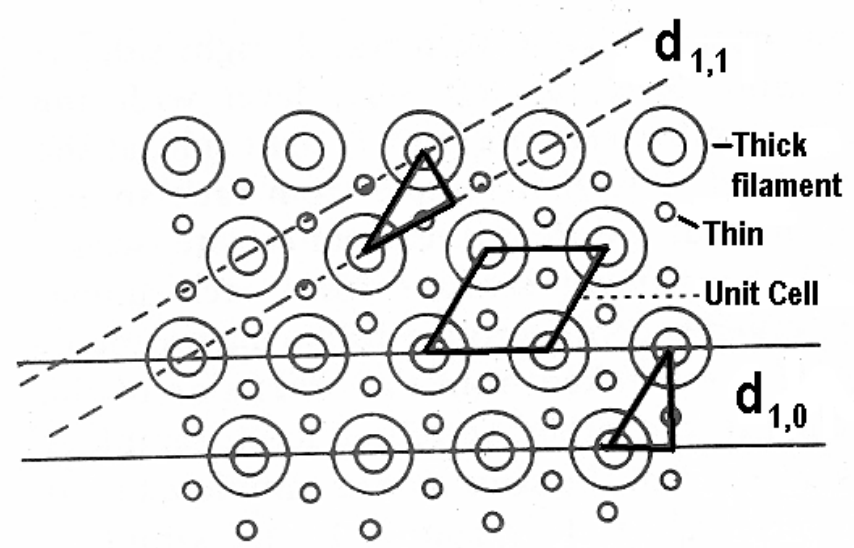

Fig. 7. X-ray diffraction shows the decreasing lateral separation of thick and thin filaments during post-mortem metabolism. From Bragg's equation, the $\mathrm{d}_{1,0}$ spacing of the unit cell is closer to the $\mathrm{x}$-ray axis than the $\mathrm{d}_{1,1}$ spacing.

\section{POLARIZED LIGHT}

Polarized light has tremendous potential and, in the laboratory, can reveal many commercially important features of a myosystem. Many of the components of a myosystem, such as myofibrils and connective tissue fibres, have a precise longitudinal arrangement of proteins. Thus, they are birefringent - having different refractive indices along and across their structure. Polarimetry involves measuring the rotation of plane polarized light. Starting with the effect of $p \mathrm{H}$ on myofibrillar birefringence, as reduced electrostatic repulsion brings filaments closer together, electron microscopy shows myofibrils decrease in diameter (Fig. 6). Thus, the velocity of light changes across but not along the myofibrils, and increases the optical path difference (along versus across myofibrils). For polarimetry of a myosystem, instead of measuring the rotation of plane polarized light, the intensity of light retaining its original plane of polarisation may be measured. Reflective surfaces at refractive index boundaries may maintain polarisation, but polarization can be lost by scattering.

Refractive index is defined by $\mathrm{c}$, the velocity of light in a vacuum and $\mathrm{v}$, its velocity in various components of myosystem, $\mathrm{n}=\mathrm{c} / \mathrm{v}$. Passing through myofibrils, light splits into two components at different velocities, the ordinary ray $(\mathrm{O})$ and the extraordinary ray $(E)$, with $\mathrm{O} \perp$ E. Birefringence, $\mathrm{n}_{\mathrm{E}}-\mathrm{n}_{\mathrm{O}}$, may be either negative or positive in sign.

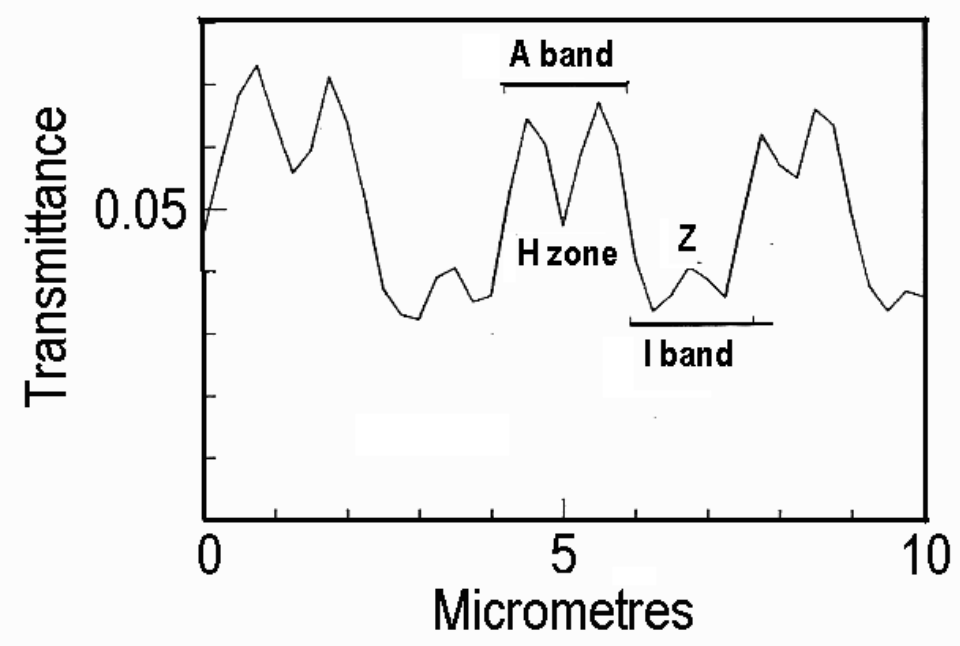

Fig. 8. Birefringence from three sarcomeres measured by scanning with a polarizing microscope.

Retardation is the decrease in velocity of light passing through a medium and may be detected as a phase retardation, the interference caused by path difference $\mathrm{E} \neq \mathrm{O}$. The path difference through a depth of myosystem $\left(\Gamma_{\mathrm{m}}\right)$ may be measured in the laboratory by ellipsometry using a de Sénarmont compensator,

$$
\Gamma_{\mathrm{m}} \mathrm{nm}=\mathrm{K}_{\lambda} \mathrm{nm} / \text { degree } \cdot \mathrm{u}^{\mathrm{o}}
$$


where $\mathrm{u}$ is the angle in degrees required for compensation, and $\mathrm{K}_{\lambda}$ is the de Sénarmont constant or path difference for $1^{\circ}$ of rotation (Fig. 9).

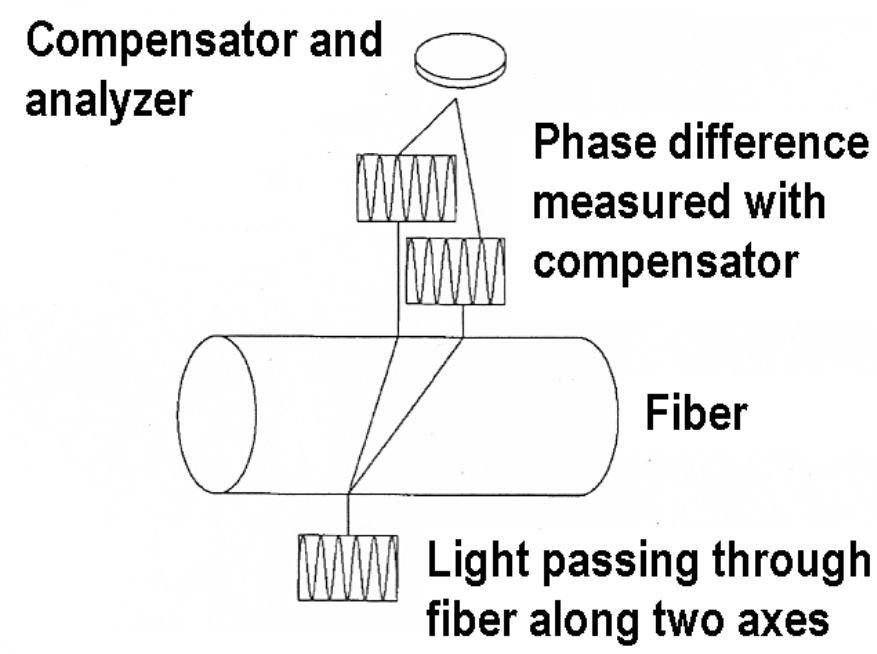

Fig. 9. Measurement of birefringence through a single muscle fiber using a De Sénarmont compensator in a polarizing microscope.

The path difference increases as $\mathrm{pH}$ decreases post-mortem towards the isoelectric point (Fig. 10). This is why polarized light is being given so much emphasis here. There is no hope of using electron microscopy or x-ray diffraction in bulk myosystems, but the changes they detect can also be detected with polarized light in a bulk myosystem.

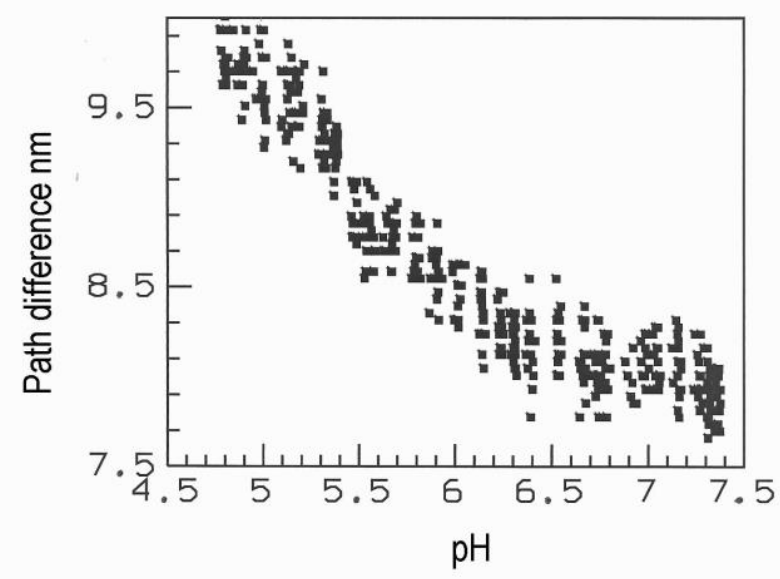

Fig. 10. As $p \mathrm{H}$ decreases post-mortem, the myofibrillar path difference increases so that more light is refracted, scattering increases and the myosystem becomes more pale.

The change in myofibrillar path difference with $\mathrm{pH}$ follows a similar pattern to that of the increase in paleness occurring post-mortem in a myosystem, and refractive scattering is probably a major source of this paleness. One would normally expect extremes of post-mortem glycolysis to be curtailed by deactivation of glycolytic enzymes. However, if the $p \mathrm{H}$ decreases below the isoelectric point, then the path difference through myofibrils decreases, refractive scattering through myofibrils decreases, and the myosystem appears darker again. This may occur in processed myosystems (ham, sausages, etc) where fermentation increases the normal decline in $p \mathrm{H}$. At very low $p \mathrm{H}$ values for a myosystem, precipitation of sarcoplasmic proteins occurs. But acid-denaturation of proteins is likely to be temperature dependent and particularly severe in a hot carcass immediately postmortem because glycolysis is exothermic. Thus, a carcass with a slow but extended decline in $p \mathrm{H}$ might not have such pale 
myosystem as would be expected at its low ultimate $p \mathrm{H}$. This explains why ultimate $p \mathrm{H}$ is not as useful as $p \mathrm{H}$ at 45 minutes post-mortem for the industrial prediction of pork colour.

Along the length of muscle fibers, there are A (anisotropic) and I (isotropic) bands matching the distribution of thick and thin filaments, respectively. At the midlength of the A band in a relaxed fiber, the otherwise strong birefringence of the A band is slightly weaker in the $\mathrm{H}$ zone, between the ends of thin filaments. Similarly, the otherwise weak birefringence of the I band has a slightly stronger region at the midlength of the $\mathrm{I}$ band caused by the $\mathrm{Z}$ line. Birefringence of the $\mathrm{Z}$ line indicates a high degree of protein filament alignment, and weakening and structural alteration of the $\mathrm{Z}$ line contributes to the tenderisation of beef during conditioning. Given that short sarcomeres and strong $\mathrm{Z}$ lines are classical sources of myosystem toughness, the challenge is to make similar measurements in bulk myosystem. At present, this is very difficult. The main problem is depolarisation by scattering - hence the interest here in the balance between polarization and scattering.

\section{MEASUREMENTS AT A MYOSYSTEM SURFACE}

From Brewster's law, the polarization angle $(\Theta)$ of reflected rays is related to refractive index, $\mathrm{n}=\tan \Theta$, but the refractive index of myosystem fluids is quite variable. One might expect the first fluid lost from myosystem post-mortem to be sarcoplasmic in origin with a high protein content, and fluid released later from the filament lattice to have a lower protein content and refractive index. This is difficult to demonstrate experimentally because different physiological types of muscle fibers may be at different stages of fluid release, depending on neural activity.

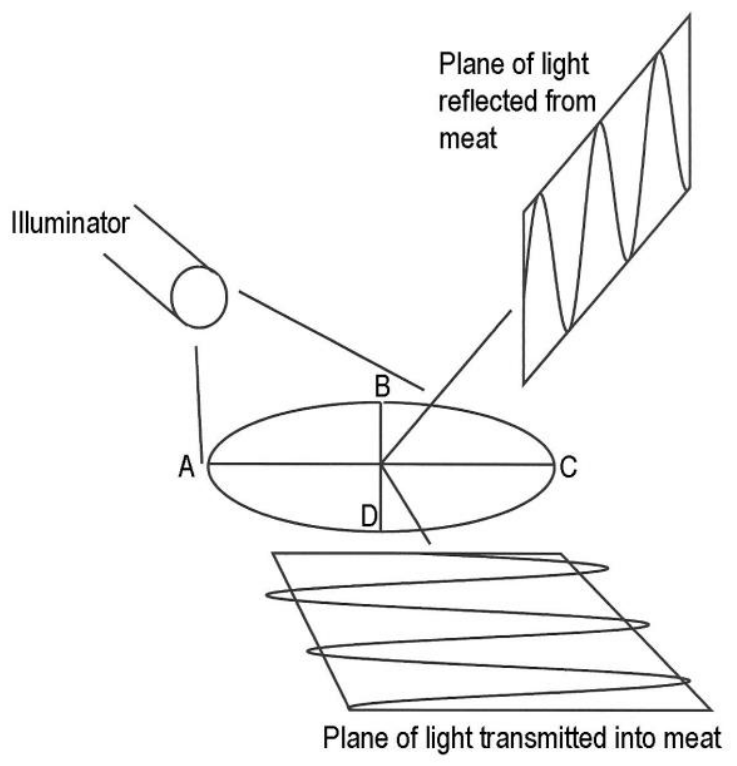

Fig. 11. Planes of polarization of light reflected from the myosystem surface versus light transmitted into the myosystem.

For an area of a myosystem illuminated to produce an ellipse (Fig. 11, ABCD), if the light from the illuminator is unpolarized and the central axis of the cone of illumination is at a fixed angle of $45^{\circ}$ to the myosystem, the reflected rays tend to be polarized primarily at $90^{\circ}$ to the plane of incidence, while the rays refracted and transmitted into the sample tend to be polarized primarily in the plane of incidence. The efficiency of polarizers and analyzers may be evaluated by measuring their extinction coefficients at different wavelengths,

$$
\mathrm{k}=\log _{10}\left(\mathrm{~T}_{0} / \mathrm{T}_{90}\right)
$$

where $T_{0}$ is with the analyzer parallel to polarizer, and $T_{90}$ is with the analyzer perpendicular to the polarizer. A similar approach may be used to find the degree to which light reflected from myosystem retains its initial polarization, using $\mathrm{R}$ rather than $\mathrm{T}$, and replacing the polarizer by the sample. Thus,

$$
\mathrm{k}=\log _{10}\left(\mathrm{R}_{0} / \mathrm{R}_{90}\right)
$$


so that a high extinction coefficient indicates a strong polarizer, and vice versa. A subscript denotes the angle of tilt at which $\mathrm{k}$ is measured. In the laboratory, with light passing through excised samples of a myosystem, there is no limit to the information that can extracted on key parameters we might wish to exploit in on-line carcass grading. But there are major obstacles for adapting these methods for use on bulk meat.

- How can we get a defined light path through the myosystem if we are limited to a myosystem surface, either from ribbing a carcass or by inserting a probe with an optical window?

- If we use a probe, how can we maintain polarization (it is lost in ordinary optical fibers and polarization-preserving fibers require precise optical alignment and are monochromatic)?

- If we use front-face polarizers on optical fibers, how can we protect them against abrasion with repeated use?

There are some solutions - yet to be fully exploited. A graded-index lens may be used to make the final connection with the carcass. These lenses are small cylinders of glass with a radial gradient in refractive index. Polarization is preserved. Parallel probes may be used, so that the optical path length through the myosystem is fixed. But there are also basic problems - yet to be fully elucidated. The birefringence signal (Fig. 10) behaves in a logical manner over a certain range - birefringence increases as sarcomeres get shorter. But, as often happens in severely cold-shortened beef, once the thick filament reaches the $\mathrm{Z}$ line it can pass through it. Then the neat lateral arrangement of filaments is lost, and birefringence starts to decrease. Thus, both seriously cold-shortened sarcomeres (very tough myosystem) and sarcomeres stretched to their maximum (very tender myosystem) give a weak birefringence signal. And there is another contradictory response from sarcomere length versus scattering.

\section{SARCOMERE LENGTH versus SCATTERING}

Sarcomere length has a strong effect on myosystem toughness and any method for on-line measurement would be a tremendous asset in carcass grading (short sarcomeres = tough meat). However, the problems involved in using light scattering to find sarcomere length are formidable. Rapid post-mortem refrigeration causes unrestrained muscles to contract, and the increased overlap of thick and thin filaments when rigor mortis develops causes toughness. Muscles cooled slowly or stretched have little filament overlap and are tender. Carcasses lacking adipose insulation, with a high surface to volume ratio, or first into an empty meat cooler have the highest risk of cold shortening. Myosystems with short sarcomeres tends to have a high degree of scattering and, hence, may appear pale. Fig. 12 shows a correlation of reflectance with sarcomere length in beef short sarcomeres increase birefringence and light scattering so the correlation is negative. A major problem is that beef with a rapid rate of post-mortem glycolysis tends to be tender because of enhanced proteolysis - and rapid glycolysis also tends to make myosystem pale by increasing light scattering. Thus, paleness or a high degree of light scattering may indicate toughness if it is caused by short sarcomeres but also tenderness if it is caused by rapid glycolysis. This has caused considerable confusion in the scientific literature. Researchers who have avoided cold shortening but who have high variance in rates of glycolysis in their experimental samples have found that tender myosystem tends to be pale, researchers who have allowed cold shortening but who have uniform rates of glycolysis in their samples have found tough myosystem tends to be pale, and researchers who have samples with a high variance in both sarcomere length and rate of glycolysis have found no relationship between paleness or light scattering and myosystem tenderness. Everything depends on the nature of the samples and when they are tested because both cold-shortening and glycolysis may show both diurnal and seasonal cycles. The problem occurs regardless of the level of instrumental complexity, and may appear with the simplest hand-held colorimeter as well as with complex methods of VIA. 


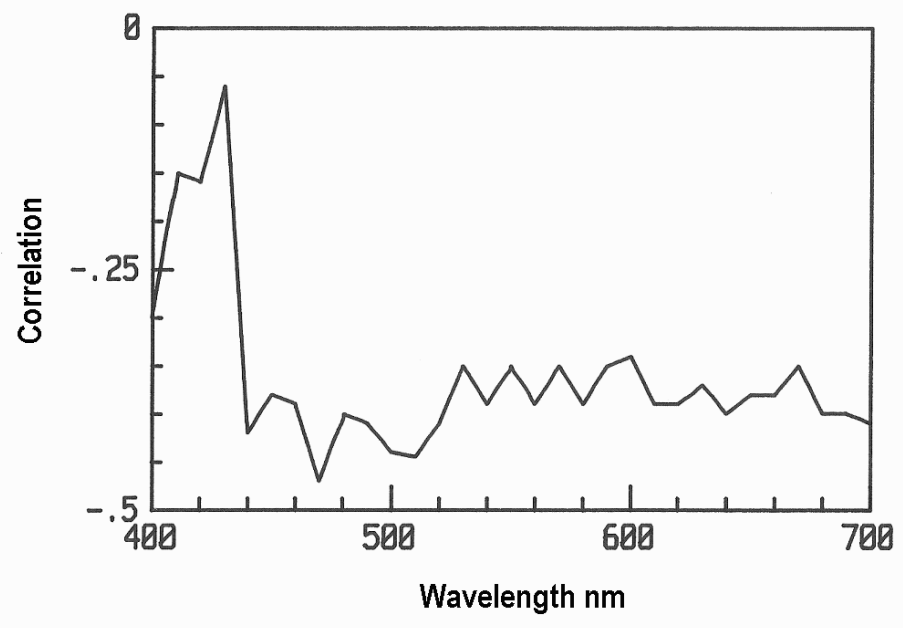

Fig. 12. Correlation of fiber-optic reflectance with sarcomere length in beef.

In Fig. 12, the correlations are weak around the Soret absorbance band at $430 \mathrm{~nm}$ where small changes in hemoproteins are a source of variance, but fairly even across the remainder of the spectrum. The relationship is not strong enough to allow sarcomere length to be predicted directly but quite likely explains how meat quality may sometimes be predicted from digital images. In summary: two strong factors affecting beef tenderness act in opposite directions on light scattering: if beef is tough because of a high $\mathrm{pH}$ it will be dark with low reflectance, but if beef is tough because of short sarcomeres it will be pale with high reflectance. Thus, if the range in $\mathrm{pH}$ is low while the range in sarcomere length is high, pale beef may be tougher than dark beef. But if the range in $\mathrm{pH}$ is high while the range in sarcomere length is low then pale beef may be more tender than dark beef.

\section{EFFECT OF SUBSURFACE OPTICAL PATHWAYS ON COLORIMETRY}

After this explanation of my personal understanding of surface and subsurface optical pathways in food myosystems, it is time to extrapolate into a totally neglected area. To what extent do subsurface optical pathways affect chromaticity coordinates?

\section{Angle of measurement}

A cube of pork $(3-\mathrm{cm})$ was illuminated on one face using a xenon arc to get a useful intensity of light at low wavelengths. The illumination was perpendicular to the long axes of muscle fibers. Light was collected from within the cube of pork using seven optical fibers mounted in hypodermic needles arranged in a radial pattern with $15^{\circ}$ angular separation (Figure 13). Thus, optical fiber 1 at $0^{\circ}$ collected light that had been transmitted across the long axes of muscle fibers while optical fiber 7 at $90^{\circ}$ collected light scattered down the long axes of muscle fibers. The apparatus was standardized (transmittance $=1$ at each wavelength and at each angle) with no sample in place. The photometric optical fibers were connected in turn to a grating monochromator and a photomultiplier. 


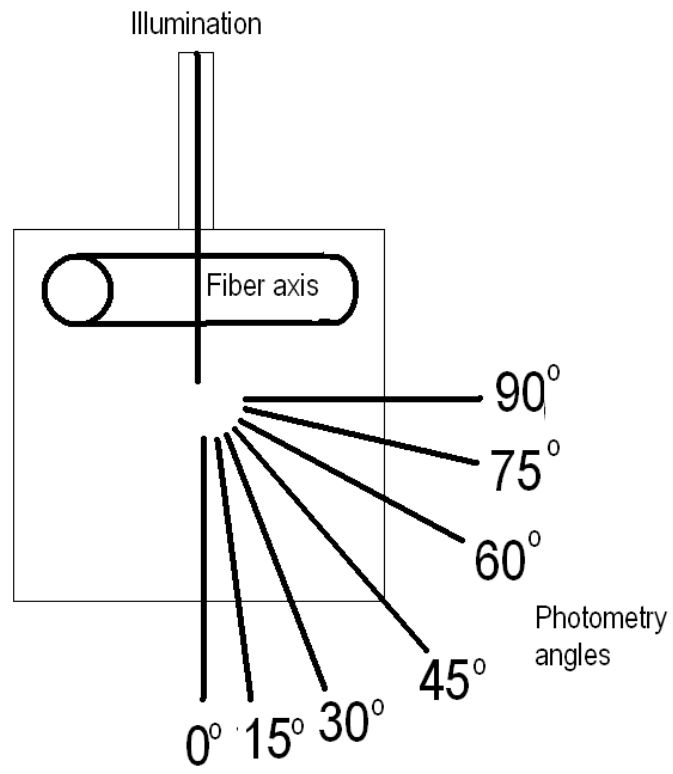

Fig. 13. Illumination perpendicular to the long axes of muscle fibers $\left(0^{\circ}\right)$ and photometry at angles from 0 to $90^{\circ}$

The photometry angle did not have much effect on CIE x $(\mathrm{r}=0.11)$, but a strong effect on CIE y $(\mathrm{r}=0.84, \mathrm{P}<0.01)$ and an even stronger effect on CIE Y\% $(r=0.95, \mathrm{P}<0.005)$. This was caused by the difference in transmittance at $0^{\circ}$ versus scattering at $90^{\circ}$. Light at long wavelengths dominated transmittance while light at short wavelengths dominated scattering, hence, the $400 / 700 \mathrm{~nm}$ ratio was dependent on the photometry angle (Fig. 14).

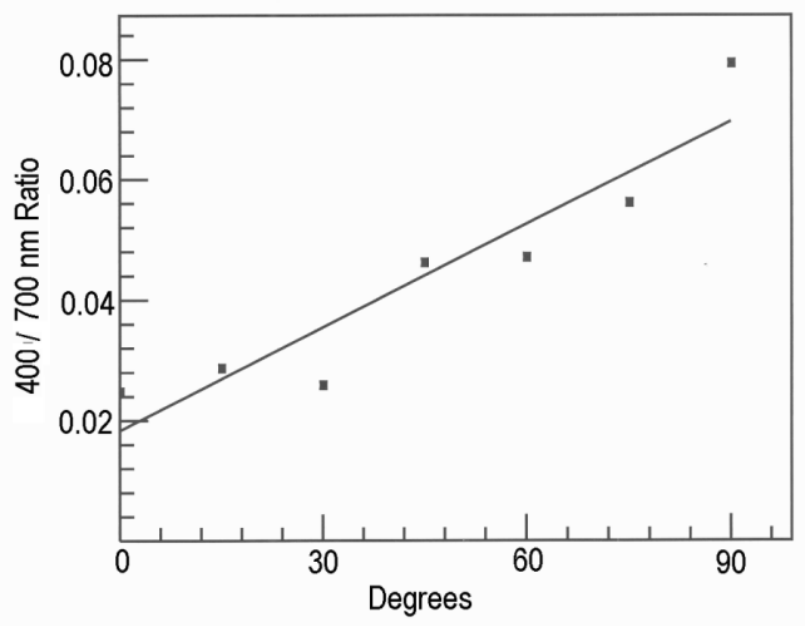

Fig. 14. Effect of photometry angle on the $400 / 700 \mathrm{~nm}$ ratio.

This makes sense from what has been deduced from numerous experiments using polarized light ellipsometry and xray diffraction. When light passes perpendicularly across the long axes of muscle fibers it passes across muscle fibers, myofibrils and myofilaments. When the $\mathrm{pH}$ of muscle drops post-mortem from the lactate produced by anaerobic glycolysis, myofilaments move closer together laterally, myofibrils shrink laterally, and the refractive index of myofibrils and muscle fibers increases. But what is the effect on CIE chromaticity coordinates? It is statistically significant $(\mathrm{P}<0.01)$ but would hardly show on a commercial colorimeter.

\section{Longitudinal scattering}

The breakthrough in explaining longitudinal optics along muscle fibers came recently from understanding the occasional iridescence of muscle fibers in transverse sections across numerous food myosystems including tuna fish (Fig. 15), roast beef and venison. When transverse sections of an iridescent myosystem are viewed with a reflecting microscope, individual muscle fibers may appear with a metallic hue. Sometimes adjacent fibers have the same hue, but often there is 
a patchwork of different hues - clearly showing individual muscle fibers act like optical fibers with minimal cross talk. The hues are multilayer interference colors created by reflective A bands with a high refractive index (Fig. 3). When only one or a small number of A bands is involved, a bright metallic color is produced. When several A bands are involved the hues produced have a pastel color. When numerous A bands are involved, the hues are lost amid clouded scattering. Thus, light scattering along muscle fibers is dominated by multilayer A band interference.

In early research on muscle fiber iridescence, fibers were grouped subjectively into different metallic colors and then analyzed spectrophotometrically to plot their interference peaks. To go beyond this, measurements were made underwater, to remove the possibility of surface diffraction gratings, and a signal processing algorithm was used to quantify interference peaks. The number of interference peaks was correlated with CIE Y\% $(r=0.51, P<0.001)$. As the number of peaks increased, the distance from the central white of the CIE chart decreased $(r=-0.52, P<0.001)$. Myofibers with low scattering had fewer interference peaks $(2.9 \pm 0.3, n=10)$ than myofibers with high scattering $(4.9 \pm 1.3, n=31, P<0.001)$. This showed the number of reflecting and interfering layers was important in relating light scattering along myofibers to surface iridescence. One or a few reflective layers produced strong interference colors while many layers produced colorless scattering. In other words, the rare metallic interference colors of iridescent myosystems are at one end of the range of light scattering along muscle fibers - in most cases for non-iridescent myosystems, multilayer interference produces a colorless, diffuse reflectance as in point B in Fig. 16 [11].

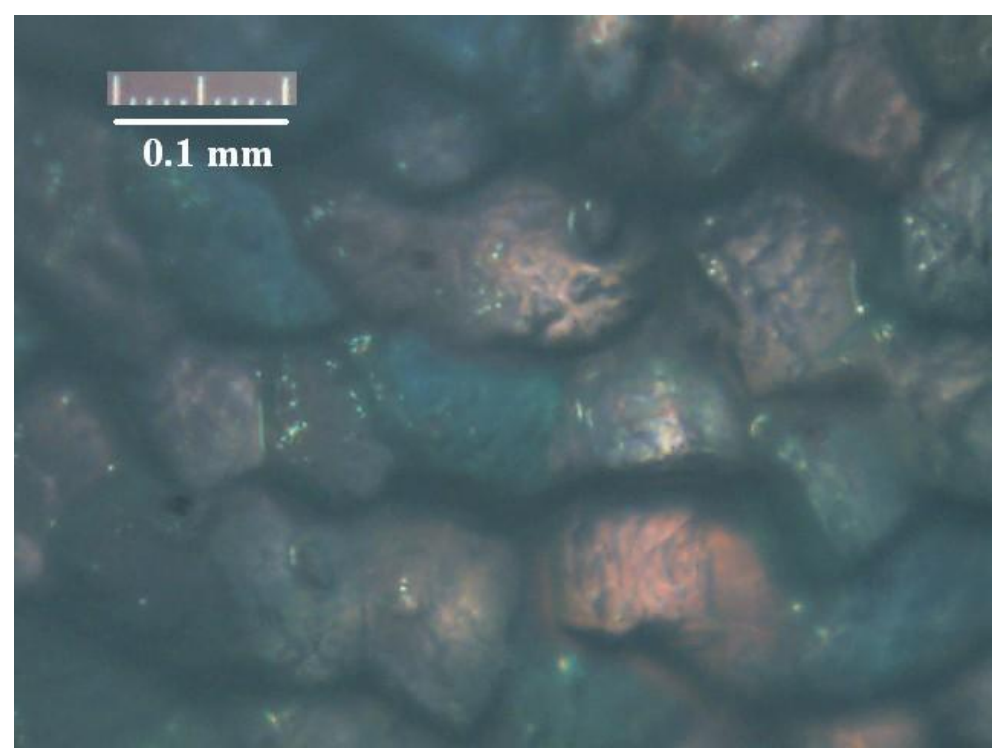

Fig. 15. Iridescence in muscle fibers of yellowfin tuna (Thunnus albacares). 


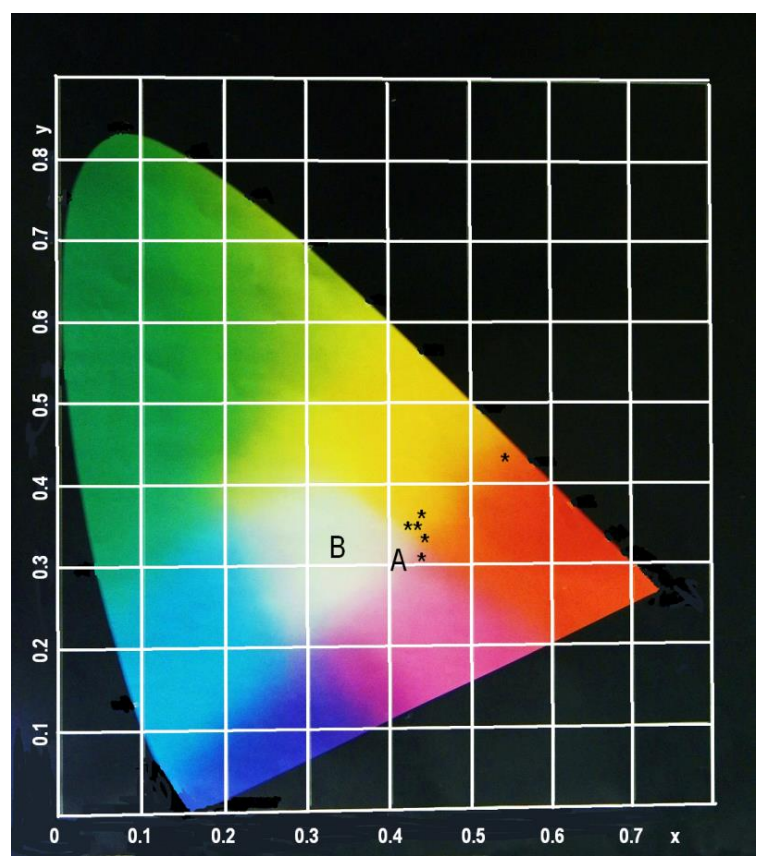

Fig. 16. CIE chromaticity coordinates of iridescent muscle fibers in cooked roast beef. The symbol * shows a few coordinates for fibers with single interference peaks, A shows the mean value for fibers with single interference peaks, while B shows the mean value for fibers with multiple peaks.

\section{Colorimetry through internal pathways}

In many colorimeters the illumination is directed at $45^{\circ}$ to the sample surface and photometry is at $90^{\circ}$ to the sample surface to minimize gloss or specular reflectance from the sample surface. But with fiber optic probes penetrating a myosystem, many other configurations are possible. These produce major changes in chromaticity coordinates as shown in Fig. 17.

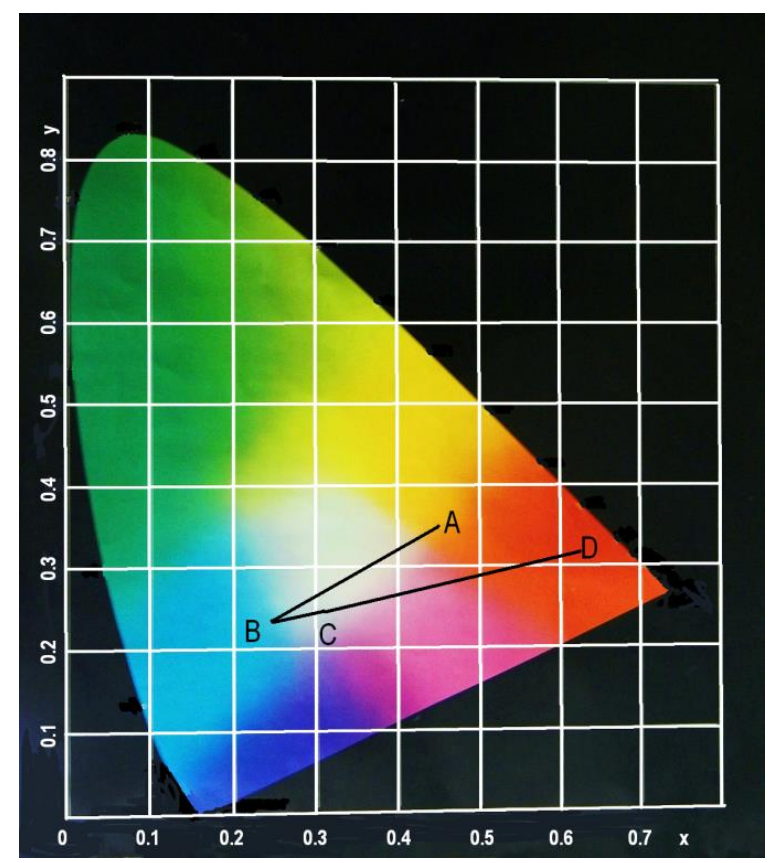

Fig. 17. Optical pathways through venison with lateral $45^{\circ}$ illumination (A), both illumination and photometry at $90^{\circ}$ to the surface (B), both illumination and photometry at $90^{\circ}$ to the surface but blocking Fresnel reflectance with crossed polarizing filters $(\mathrm{C})$, and with light transmitted through the sample (D). Different pathways are highly significant $(\mathrm{P}<$ $0.001)$. 
The interesting thing about light transmitted through a low myoglobin myosystem such as pork when tested with fiber optics is that it is a linear arithmetic function of path length, not an exponential relationship [12]. Thus, parallel needles containing optical fibers may be used for both optical measurements of light scattering and electrical measurements of membrane capacitance to predict pork quality [13].

\section{CONCLUSIONS}

Doubtless there are many differing opinions on the basic factors affecting the colorimetry of food myosystems. The author's opinion is that the optical complexity of apparatus is inversely related to the certainty of what is being measured. From laser confocal studies to hyperspectral imaging it may well be possible to establish empirical correlations with the commercial properties of a myosystem, but explaining the basis of the correlations requires an inductive scientific approach rather than an empirical approach. The history of materials science tells us that the bulk properties of materials ultimately depend on their microstructure. Thus, to understand the scientific basis of the bulk colorimetry of myosystems we must delve into microcolorimetry [14]. Using the well understood methods of x-ray diffraction and electron and light microscopy looks to this author like a safe basis for future inductive research.

\section{REFERENCES}

[1] Fox, J.B. (1987). The pigments of meat. In The Science of Meat and Meat Products J.F. Price and B.S. Schweigert (Eds). pp. 193-216. Food Nutrition Press, Westport, CT. https://agris.fao.org/agris-search/search.do?recordID=US891288

[2] Swatland, H.J. (1989). Effect of temperature $\left(0-80^{\circ} \mathrm{C}\right)$ on the interior reflectance of ovine sternomandibularis muscle. International Journal of Food Science and Technology 24:503-510. https://ifst.onlinelibrary.wiley.com/doi/abs/10.1111/j.1365-2621.1989.tb00673.x

[3] Bendall, J.R. and Swatland, H.J. (1988). A review of the relationships of pH with physical aspects of pork quality. Meat Science 24:85-126. https://www.sciencedirect.com/science/article/abs/pii/0309174088900526

[4] Elliott, R.J. (1967) Effect of optical systems and sample preparation on the visible reflection spectra of pork. Journal of the Science of Food and Agriculture 18: 332- 338. https://doi.org/10.1002/jsfa.2740180802

[5] Swatland, H.J. (2011). Basic science for carcass grading. VI Congresso Brasileiro de Ciência e Tecnologia de Carnes. pp. 119-126. VI Congresso Brasileiro de Ciência e Tecnologia de Carnes (suinoculturaindustrial.com.br)

[6] Ramanathan, R., Hunt, M.C., Mancini, R.A., Nair, M.N., Denzer, M.L., Suman, S.P., and Mafi, G.G. (2020). Recent updates in meat color research: integrating traditional and high-throughput approaches. Meat and Muscle Biology 4(2). https://doi.org/10.22175/mmb.9598

[7] Hughes, J.M., Clarke, F.M., Purslow, P., and Warner, R.D. (2019). Meat color is determined not only by chromatic heme pigments but also by the physical structure and achromatic light scattering properties of the muscle: muscle light scattering properties. Comprehensive Reviews in Food Science and Food Safety 19(1) December 2019. DOI: 10.1111/15414337.12509

[8] Billmeyer, F.W. and Saltzman, M. (1981). Principles of Color Technology. John Wiley, New York.

[9] Bowman, W. 1840. On the minute structure and movements of voluntary muscle. Philosophical Transactions 130: 457501. https://royalsocietypublishing.org/doi/10.1098/rstl.1840.0022

[10] Ruedt, C., Gibis, M., Barbut, S. and Weiss, J. (2021). Colour changes with longitudinal compression supports hypothesis of multilayer interference as a cause of meat iridescence. International Journal of Food Science and Technology 56: 250-258. https://ifst.onlinelibrary.wiley.com/doi/epdf/10.1111/ijfs.14626

[11] Swatland, H.J. (2019). Signal analysis of optical interference in relation to colorimetry for measurements made along individual myofibers in cooked beef. Asian Journal of Agriculture and Food Sciences 7 (6). Https://www.ajouronline.com/index.php/AJAFS/article/view/5951

[12] Swatland, H.J. (1999). Photometric relationship between path length and transmittance through pork measured by $\begin{array}{llllll}\text { fiber optics. } & \text { Food } & \text { Research } & \text { International } & 32: & 305-312 .\end{array}$ https://www.sciencedirect.com/science/article/abs/pii/S096399699900085 
[13] Swatland, H.J. (1997). Post-mortem changes in pork using parallel needles for both light scattering and low-frequency $\begin{array}{lccc}\text { electrical } & \text { properties. } & \text { Food } & \text { Research } \\ \text { https://www.sciencedirect.com/science/article/abs/pii/S0963996997000495 }\end{array}$

[14] Swatland, H.J. (2017). A review of microcolorimetry for textile, food, dental and optoelectronic industries. Asian Journal of Engineering and Technology 5:140-151. https://www.ajouronline.com/index.php/AJET/view/5065 